\title{
Erratum: Non-standard neutrino interactions and low energy experiments
}

\author{
Wolfgang Altmannshofer, ${ }^{a}$ Michele Tammaro $^{b}$ and Jure Zupan ${ }^{b}$ \\ ${ }^{a}$ Santa Cruz Institute for Particle Physics, \\ Santa Cruz, CA 95064, U.S.A. \\ ${ }^{b}$ Department of Physics, University of Cincinnati, \\ Cincinnati, Ohio 45221, U.S.A. \\ E-mail: waltmann@ucsc.edu, tammarme@mail.uc.edu, zupanje@ucmail.uc.edu
}

ERRATUM TO: JHEP09(2019)083

ABSTRACT: We correct the constraints on non-standard neutrino interactions (NSI) from deep inelastic scattering. We also provide a detailed derivation of the Borexino bounds on Rayleigh operators, while the original manuscript relied on a naive estimate. The updated Borexino constraints are weaker than the ones presented in the original manuscript.

ArXiv EPRINT: 1812.02778

\subsection{Constraints on NSI from deep inelastic scattering}

The bounds listed in equations (6.22) and (6.23) of section 6.3 of the original manuscript [1] contain typographical errors. The correct bounds are

$$
\begin{array}{lllll}
\mathcal{C}_{1, u(d, s)}^{(6)}: & \Lambda>669(713,275) \mathrm{GeV} & \left(\nu_{e}\right), & \Lambda>1402(1540,780) \mathrm{GeV} & \left(\nu_{\mu}\right), \\
\mathcal{C}_{2, u(d, s)}^{(6)}: & \Lambda>419(451,322) \mathrm{GeV} & \left(\nu_{e}\right), & \Lambda>1048(628,472) \mathrm{GeV} & \left(\nu_{\mu}\right)
\end{array}
$$

\subsection{Other constraints}

The second part of section 6.4 of the original manuscript [1], which describes the bounds on Rayleigh operators from Borexino, relied on a naive estimate that led to an overestimate of the bounds. The paragraph after equation (6.30), up to and including equation (6.32), should be replaced with the text below: 
The measured neutrino scattering rates in Borexino also bound Rayleigh operators $\mathcal{C}_{1,2}^{(7)}$. These contribute at 1-loop to the $\nu e \rightarrow \nu e$ scattering and at tree level to $\nu e \rightarrow \nu e \gamma$, i.e., to the neutrino scattering with an emission of an extra photon. In Borexino both processes result in the same detector response and thus need to be added, giving the total scattering cross section $\sigma_{\nu_{\alpha}}$ for neutrino flavor $\nu_{\alpha}$ (defined to include also the SM contributions). We calculate the tree-level $\nu e \rightarrow \nu e \gamma$ scattering numerically by implementing the Rayleigh operator in MadGraph [2]. For the much smaller 1-loop induced $\nu e \rightarrow \nu e$ scattering we use a simple NDA estimate, taking $\sigma \simeq\left(\left|\hat{\mathcal{C}}_{1}^{(7)}\right|^{2}+3\left|\hat{\mathcal{C}}_{2}^{(7)}\right|^{2} / 2\right) \alpha^{4} s^{2} /\left(48 \pi^{4}\right)$, where $s=m_{e}^{2}+2 m_{e} E_{\nu}$ is the center of mass energy of the scattering process.

The event rate per day per 100 tons of detector is given by

$$
\begin{aligned}
R_{i}\left(\hat{C}_{1}^{(7)}, \hat{C}_{2}^{(7)}\right)=T N_{e} \int d E_{\nu} \phi_{i}\left(E_{\nu}\right)[ & P_{i}^{e} \sigma_{\nu_{e} e}\left(E_{\nu}, \hat{C}_{1, e}^{(7)}, \hat{C}_{2, e}^{(7)}\right) \\
& \left.+P_{i}^{\mu} \sigma_{\nu_{\mu} e}\left(E_{\nu}, \hat{C}_{1, \mu}^{(7)}, \hat{C}_{2, \mu}^{(7)}\right)\right],
\end{aligned}
$$

with $T=1$ day $=8.64 \cdot 10^{4} \mathrm{~s}$ the exposure time and $N_{e}=3.307 \cdot 10^{31}$ the number of target electrons in 100 tons of detector mass, and $\ell=e, \mu$ denote the incoming neutrino flavor for the $C_{j, \ell}^{(7)}, j=1,2$ Wilson coefficients. The main components of the solar neutrino flux on Earth, $\phi_{i}\left(E_{\nu}\right)$, ref. [3], are due to proton-proton fusion $(i=p p)$, Berillium 7 electron capture $\left(i={ }^{7} \mathrm{Be}\right)$, and proton electron capture $(i=p e p)$. The $\nu_{e}$ from $p p$ have a continuous energy spectrum with the maximal energy $E_{\nu, \max }=$ $0.423 \mathrm{MeV}$, while ${ }^{7} \mathrm{Be}$ and pep neutrinos have an almost monochromatic spectrum with energy $E_{7 \mathrm{Be}}=0.863 \mathrm{MeV}$ and $E_{p e p}=1.445 \mathrm{MeV}$. The lower integration limit $E_{\nu, \min }=0.139 \mathrm{MeV}$ in (6.31) is the minimum incoming neutrino energy that can still produce $T=T_{t h} \sim 50 \mathrm{keV}$ recoil in Borexino, while the upper integration limit is $E_{\nu, \max }$. The $\nu_{e}$ produced in the Sun undergo flavor oscillations while propagating to Earth, giving the $\nu_{e}$ survival probabilities $P_{p p}^{e}=0.554, P_{7_{\mathrm{Be}}}^{e}=0.536, P_{p e p}^{e}=0.529$, once matter effects are taken into account [4], while $P_{i}^{\mu}=\left(1-P_{i}^{e}\right) / 2$, assuming for simplicity maximal $\theta_{23}$.

We derive the bounds on the experimentally allowed values of the Rayleigh operators $\hat{C}_{j, \ell}^{(7)}$ from the chi-squared function

$$
\chi^{2}\left(\vec{\alpha}, \hat{C}_{j}\right)=\sum_{i} \frac{\left[R_{\mathrm{meas}, i}-R_{i}\left(\hat{C}_{1}^{(7)}, \hat{C}_{2}^{(7)}\right)\left(1+\alpha_{i}\right)\right]^{2}}{\sigma_{i}^{2}}+\left(\frac{\alpha_{i}}{\sigma_{\alpha_{i}}}\right)^{2}
$$

where the sum is over the three types of solar neutrino fluxes, with the corresponding $\mathrm{SM}$ rates in Borexino phase-II given by $R_{p p}(0)=131.4, R_{7_{\mathrm{Be}}}(0)=48.1, R_{p e p}(0)=2.8$. The parameters $\alpha_{i}$ are marginalized over, and account for theoretical errors on the predictions, $\sigma_{\alpha_{p p}}=1.1 \%, \sigma_{\alpha^{7} \mathrm{Be}}=5.8 \%, \sigma_{\alpha_{p e p}}=1.5 \%$, while $R_{\text {meas }, p p}=134 \pm 10$, $R_{\text {meas, }{ }^{7} \mathrm{Be}}=48.3 \pm 1.1, R_{\text {meas }, \text { pep }}=2.43 \pm 0.36$ are the masured event rates and their statistical uncertainties, $R_{\text {meas }, i} \pm \sigma_{i}$. We convert the $1 \sigma$ allowed ranges on $\hat{C}_{j, \ell}^{(7)}$ 
(switching on one at a time) to the bounds on the effective scale, $\Lambda$

$$
\begin{aligned}
& \mathcal{C}_{1}^{(7)}: \quad \Lambda>86 \mathrm{MeV} \quad\left(\nu_{e}\right), \quad \Lambda>55 \mathrm{MeV} \quad\left(\nu_{\mu}\right), \\
& \mathcal{C}_{2}^{(7)}: \quad \Lambda>98 \mathrm{MeV} \quad\left(\nu_{e}\right), \quad \Lambda>63 \mathrm{MeV} \quad\left(\nu_{\mu}\right) \text {, }
\end{aligned}
$$

by setting the corresponding dimensionless Wilson coefficient $C_{j, \ell}^{(7)}=1$ in (2.1).

Our previous estimates of the modified neutrino scattering rates in Borexino resulted in the strongest bounds on the Rayleigh operators. The corrected bounds from Borexino are substantially weaker and insignificant compared to the bounds from COHERENT and CHARM.

The corrected bounds from deep inelastic scattering and from Borexino affect the plots in figures 6 and 7 . The updated figures are shown below.
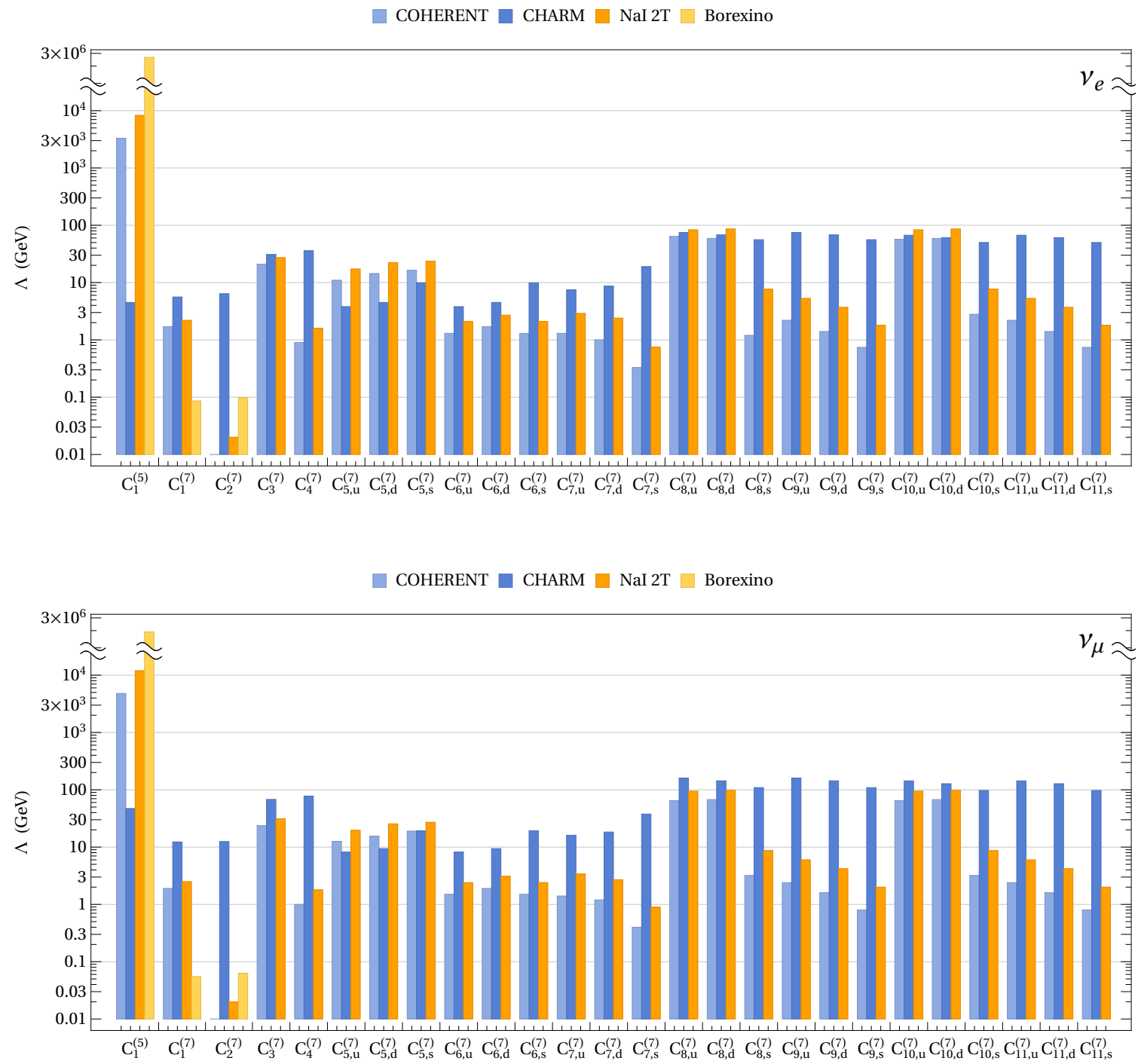

Figure 6. Limits from COHERENT, CHARM, Borexino, and projected limits from a NaI $2 \mathrm{~T}$ experiment on the scale $\Lambda$ of dimension 5 and dimension 7 NSI operators for electron neutrinos (top) and muon neutrinos (bottom). 

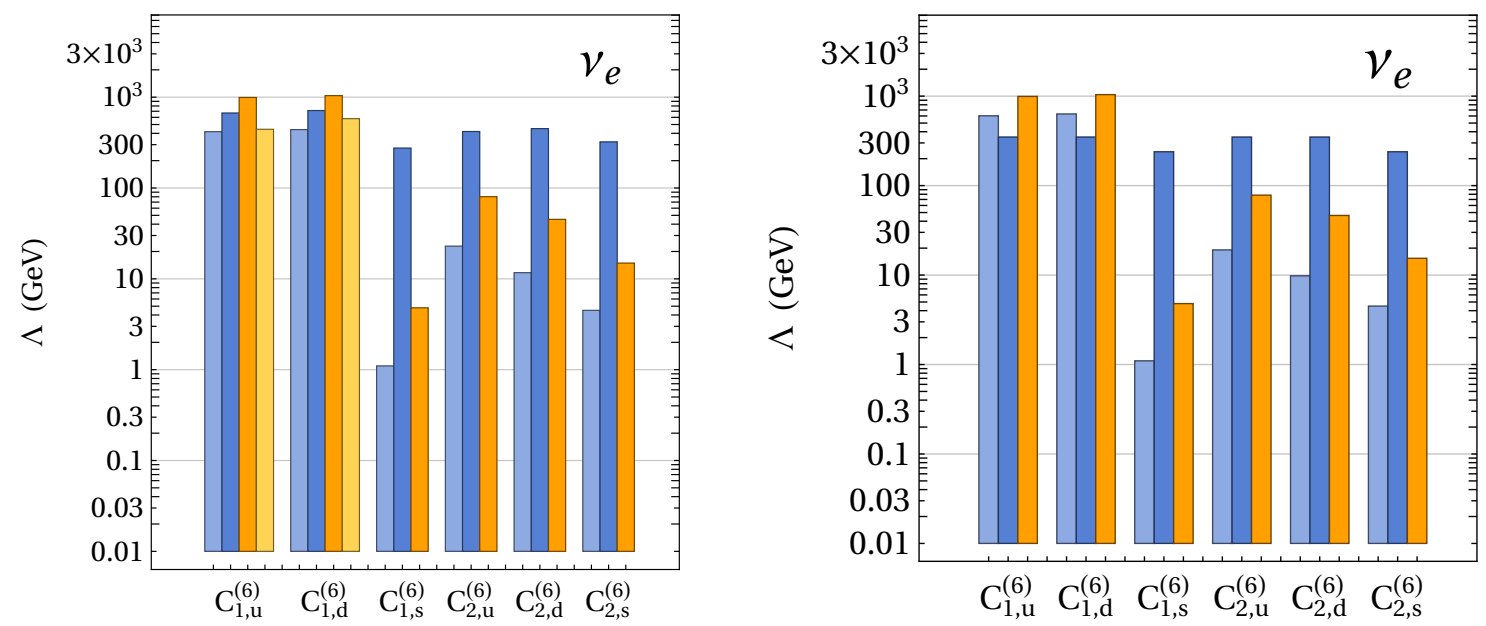

$\square$ COHERENT $\square$ CHARM $\square$ NaI 2T $\square$ oscillations
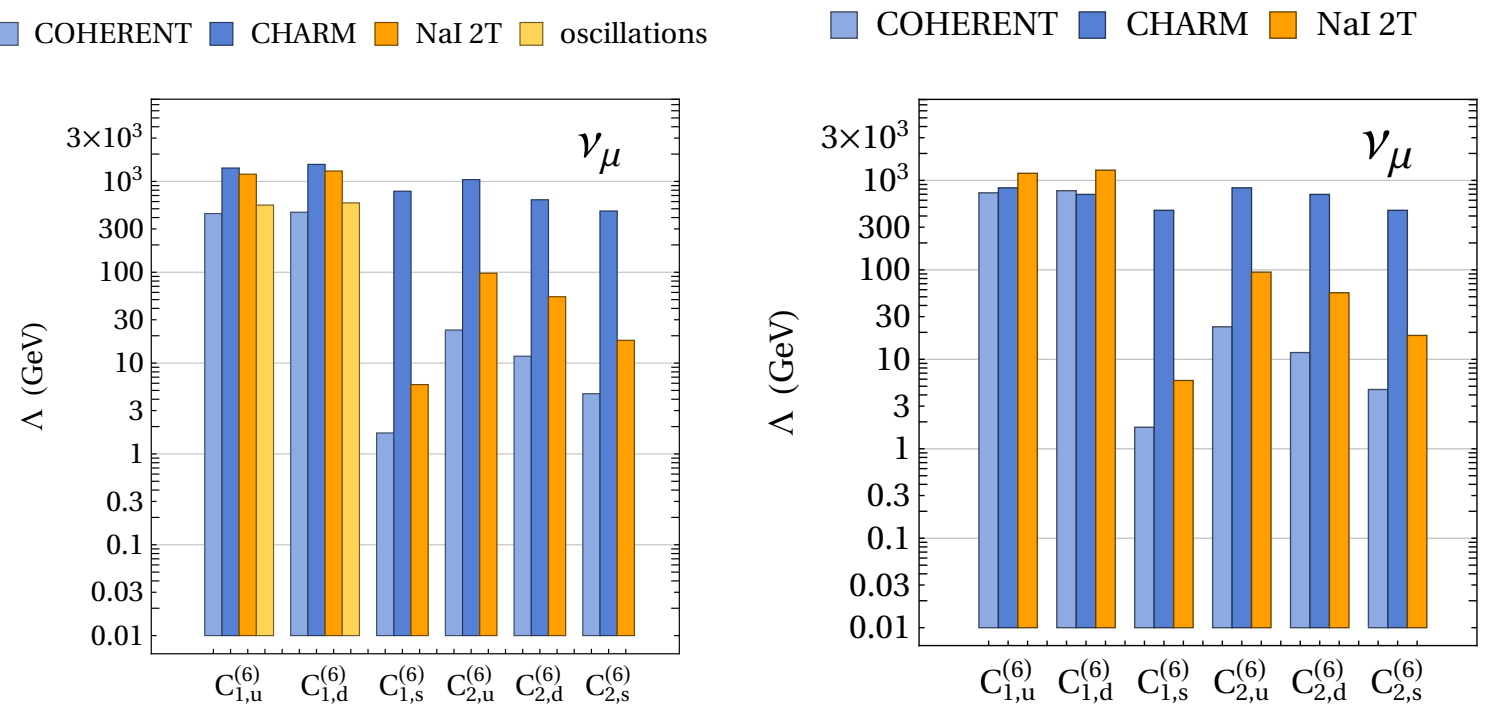

Figure 7. Limits from COHERENT, CHARM, neutrino oscillations and projected limits from a $\mathrm{NaI} 2 \mathrm{~T}$ experiment on the scale $\Lambda$ of dimension 6 operators for electron neutrinos (top) and muon neutrinos (bottom). The two panels on the left correspond to lepton flavor conserving operators $\left(\nu_{i} \rightarrow \nu_{i}\right)$, the ones on the right to lepton flavor violating operators $\left(\nu_{i} \rightarrow \nu_{j}, i \neq j\right)$. 
Open Access. This article is distributed under the terms of the Creative Commons Attribution License (CC-BY 4.0), which permits any use, distribution and reproduction in any medium, provided the original author(s) and source are credited.

\section{References}

[1] W. Altmannshofer, M. Tammaro and J. Zupan, Non-standard neutrino interactions and low energy experiments, JHEP 09 (2019) 083 [arXiv: 1812.02778] [INSPIRE].

[2] J. Alwall, M. Herquet, F. Maltoni, O. Mattelaer and T. Stelzer, MadGraph 5: Going Beyond, JHEP 06 (2011) 128 [arXiv:1106.0522] [INSPIRE].

[3] E. Vitagliano, I. Tamborra and G. Raffelt, Grand unified neutrino spectrum at Earth: Sources and spectral components, Rev. Mod. Phys. 92 (2020) 045006.

[4] A.N. Khan, W. Rodejohann and X.-J. Xu, Borexino and general neutrino interactions, Phys. Rev. D 101 (2020) 055047 [arXiv: 1906.12102] [INSPIRE]. 